\title{
HISTORICAL RAINFALL FOR URBAN STORM DRAINAGE DESIGN
}

\author{
Jasna Petrovic and Jovan Despotovic
}

Faculty of Civil Engineering, University of Belgrade, P.O. Box 895, 11000 Belgrade, Yugoslavia

\begin{abstract}
Traditional design method for urban drainage systems is based on design storms and its major drawback is that frequencies of peak flows in the system are considered equal to frequencies of design storms. An alternative is to use historical storms with rainfall-runoff models to produce a series of possible flows in the system and their frequencies. The latter approach involves more computations and can be laborious for larger catchments. This paper considers ways to reduce the set of historical storms to be involved in design procedure and yet to lead to realistic flow frequencies. Frequencies obtained by rainfall-runoff simulation at an experimental catchment are compared with frequencies of observed peak flows in the system.
\end{abstract}

\section{KEYWORDS}

Design storms; design flows; rainfall depth; rainfall intensity

\section{INTRODUCTION}

Conventional design of urban storm drainage systems involves sizing of pipes and other structures based on design flow rates. The design flows are generally obtained by applying a rainfall-runoff model to a set of design storms or historical storms. While the use of design storms implies that the probabilistic characteristics (frequency) of rainfall are transferred to design flows, the use of historical storms with rainfall-runoff models leads to a series of possible flows in the system whose frequencies are to be determined by means of statistical analysis. The latter approach involves more computations and can be laborious for larger catchments, but it is believed to yield more realistic results.

The historical storm approach has been discussed in the urban hydrological literature from the point of view of reducing the amount of computations by limiting the number of historical events that should be subjected to rainfall-runoff modelling (Harremoes et al., 1984; Packman and Kidd, 1980; Zhu et al., 1996). This paper continues this discussion and previous work by the same authors (Despotovic et al., 1996; Despotovic and Petrovic, 1996) by taking into consideration different sets of historical storms and by using them as input to a rainfall-runoff model in order to obtain corresponding peak flows and their frequencies. All computations performed are based on rainfall and runoff measurements at the experimental urban storm drainage catchment "Miljakovac 2" in Belgrade for the period 1981-1993. This means that the "true" flow frequency distribution is known, so that the frequencies of simulated flows using historical storms can be compared with "true" frequencies. In this approach, it is assumed that the distribution of observed flow frequencies is reliable (i.e. that it represents true peak flow frequencies) and that the rainfall-runoff model is appropriately calibrated for the catchment in hand.

Several different sets of historical storms are considered. First, all available historical storms are used for the rainfall-runoff simulation. Then, several subsets of historical storms are formed, in order to reduce the amount of computation. These are the historical storms with significant rainfall depth, the historical storms with significant rainfall intensity, and finally, the historical storms with duration close to the catchment's 
time of concentration. The idea is to investigate which subset of all historical storms could be used for the sewer system design and to compare these storms with the traditional design storms derived from local IDF curves.

\section{METHODOLOGY}

\section{Observed data and simulations}

The experimental urban drainage catchment "Miljakovac" was set up in early eighties in the Belgrade suburb Miljakovac (Maksimovic et al., 1986). The total area of the catchment is 25.5 ha. The southwestern part of the catchment presents a very well defined subcatchment of 7.14 ha, not only by terrain characteristics, but also by a separate branch of sewer network. Pervious areas are estimated at $62 \%$ of total area, and out of $38 \%$ of impervious areas, $52 \%$ is considered as effective impervious areas, i.e. about $20 \%$ of total area is effectively contributing to surface runoff. The catchment is equipped with two flow measuring structures at the outlets of the subcatchment and the whole catchment. Because of better consistency of flow data at the subcatchment, this paper deals only with the peak flows observed at the subcatchment outlet. Two rain gauges (a tipping-bucket and a Hellmann rain gauge) are located together within the catchment, near the subcatchment outlet.

Runoff simulations were performed with the BEMUS, a physically based rainfall-runoff model (Radojkovic and Maksimovic, 1984). The model was applied to the Miljakovac data on many occasions, especially in the model testing phase. For the purpose of this study, the model was calibrated for the medium and extreme events, so it is believed that the catchment's behaviour during significant events is described realistically by the model.

\section{Sets of "critical" historical storms}

The rainfall record spans from 1981 to 1993 and consists of about 500 historical storms. Out of the complete set, a set of 94 storms with rainfall depth greater than $5 \mathrm{~mm}$ and with intensity greater than $0.5 \mathrm{~mm} / \mathrm{min}$ was established. This set will be referred to as to the set of "all historical storms", meaning all significant storms. Figures 1 and 2 show how peak flows at the catchment outlet depend on two main rainfall characteristics rainfall depth and maximum intensity. Apart from what is clear - the greater rainfall depth and intensity the greater peak flows - an interesting relationship between maximum rainfall intensity and peak flows can be seen in Fig. 2. For a specific storm, peak flow cannot be greater than a certain value (this value obviously being a linear function of intensity), i.e. it can only be smaller (depending on antecedent conditions and other

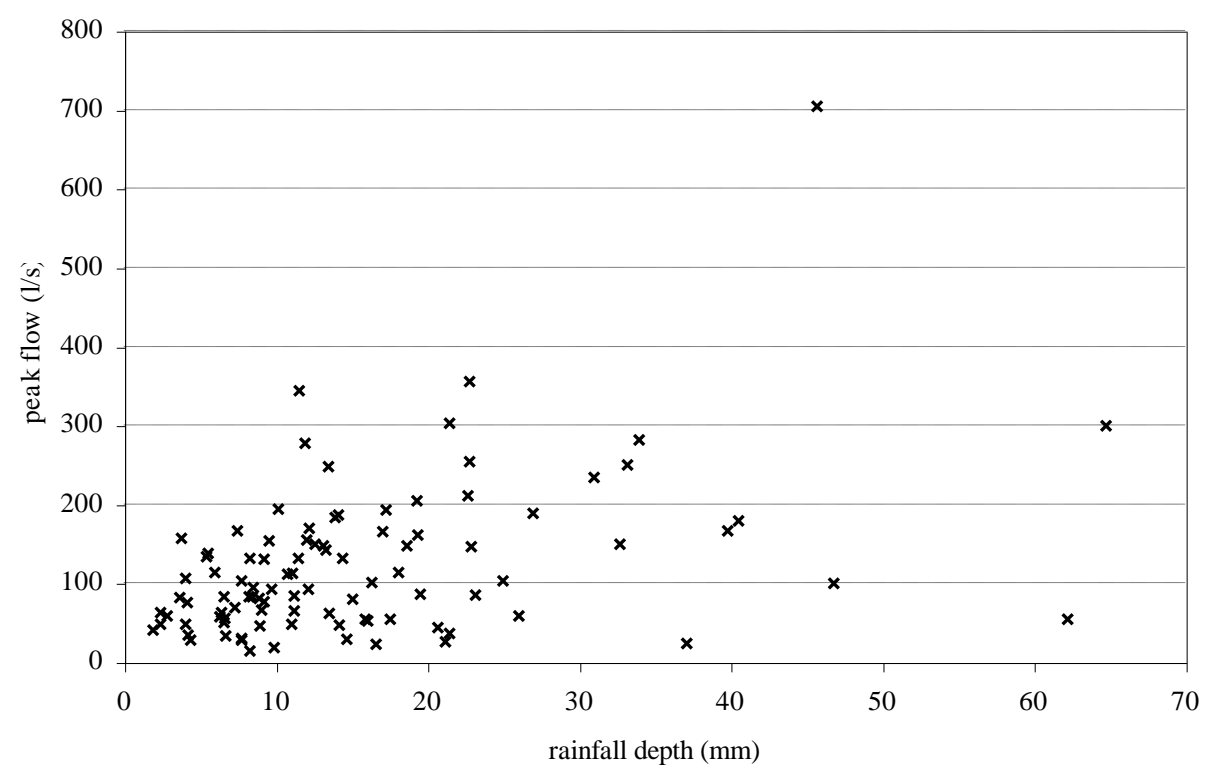

Figure 1. Peak flows vs. rainfall depth of historical storms. 


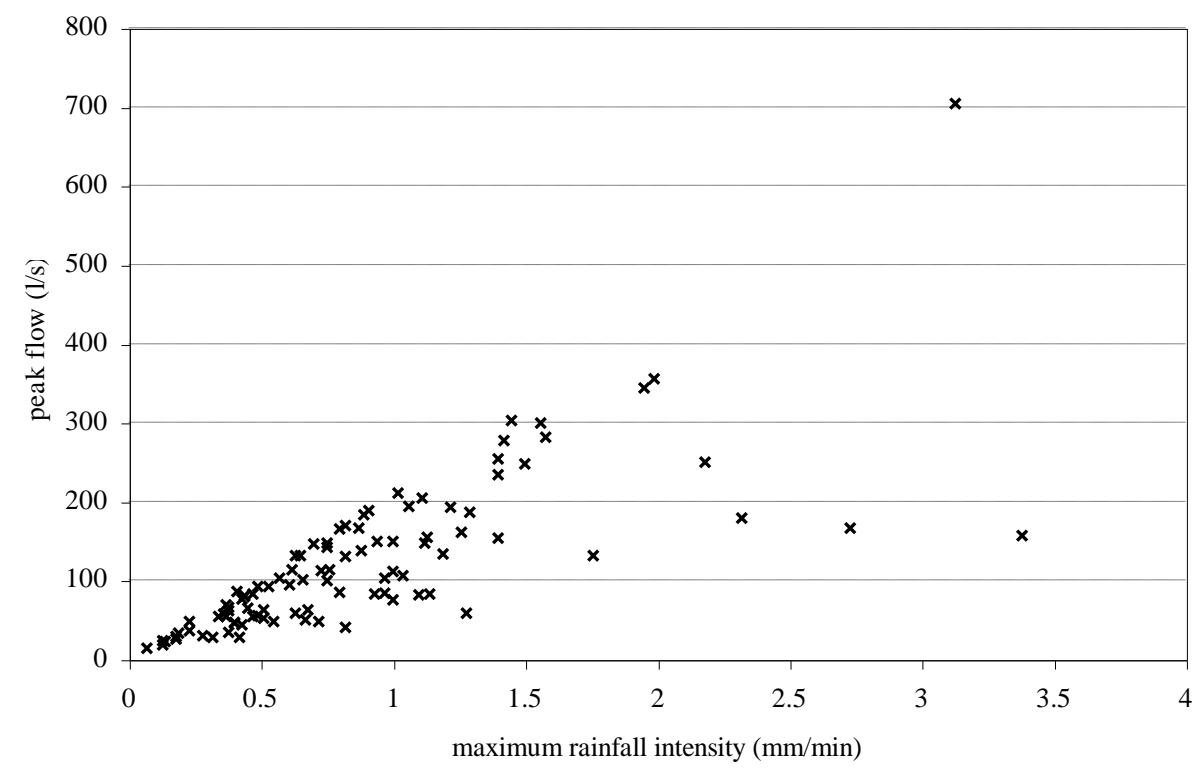

Figure 2. Peak flows vs. maximum rainfall intensity of historical storms.

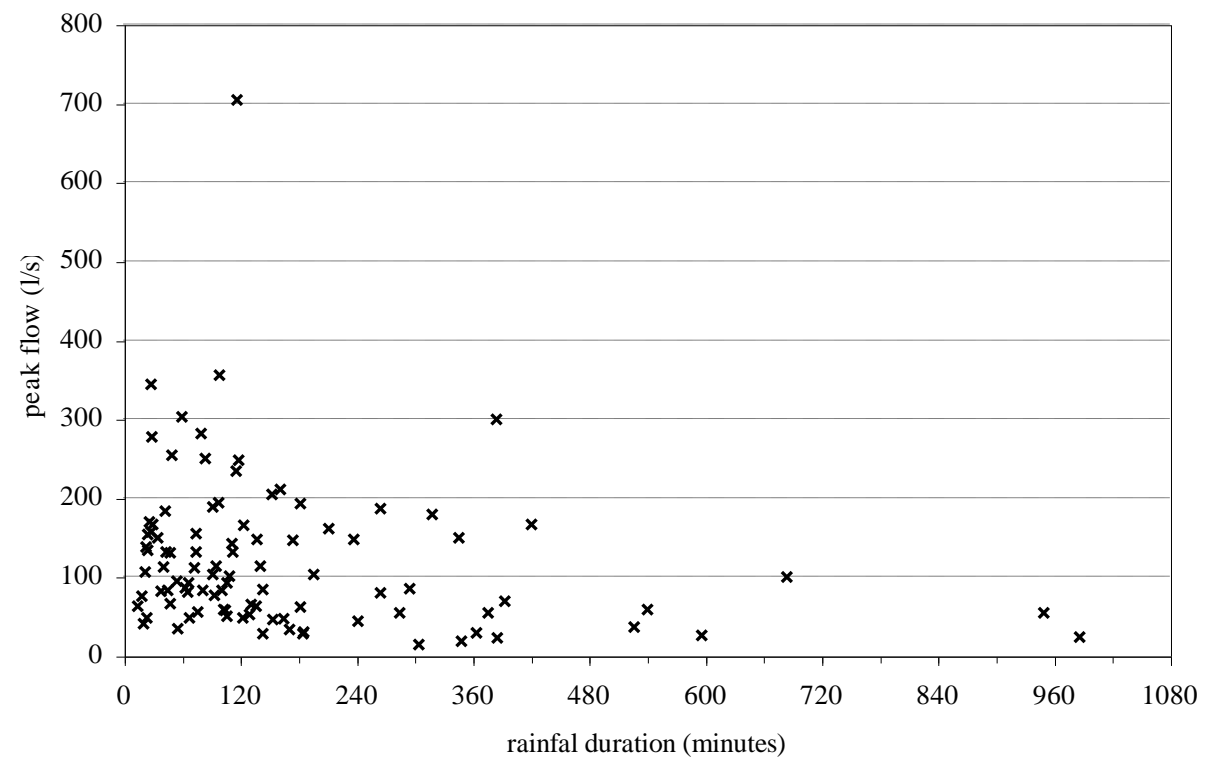

Figure 3. Peak flows vs. rainfall duration of historical storms.

factors). In other words, storms with modest maximum rainfall intensity cannot produce extreme flows. Therefore it is implied that historical storms with the highest maximum intensities could make a reliable subset of storms for design procedure.

Rainfall duration is another rainfall characteristic whose influence on peak flows should be considered, and Fig. 3 shows this relationship. In traditional design storm concept, design storm duration is chosen as the duration which gives the maximum peak flows, but generally this duration is set close or equal to the catchment's time of concentration. In the case of our experimental catchment, time of concentration is estimated between 15 and 20 minutes. It can be seen from Fig. 3 that some of the highest peak flows occurred not only for such a short rainfall duration, but also for storms with duration up to 6 hours.

In order to investigate which subset of historical storms could be used for the sewer system design, three subsets of historical storms have been identified:

- Storms with rainfall depth greater than $10 \mathrm{~mm}$ 
- Storms with rainfall intensity greater than $0.8 \mathrm{~mm} / \mathrm{min}$

- Storms with duration close to the catchment's time of concentration (duration up to 60 minutes).

All these storms were subject to rainfall-runoff simulation, and the frequencies of resulting peak flows at catchment outlet were calculated.

\section{Design storms}

The intensity-duration-frequency (IDF) curves were derived through the frequency analysis of the observed rainfall data at the Miljakovac catchment. However, these local IDF curves are based on a very short rainfall record (13 years). The nearest rain gauge station with a sufficiently long record is the station Vracar, located $6 \mathrm{~km}$ from the Miljakovac rain gauge and with 65 years of record. The major differences in the IDF curves from the two stations are in rainfall intensities for durations up to 30 minutes, where the Miljakovac data give underestimation of about $20 \%$. Therefore, the IDF curves from the Vracar station were chosen as the basis for calculation of the design storms.

Two types of design storms were considered: a block storm (constant intensity), and a temporally distributed design storm. The temporal variability of rainfall has also been analyzed statistically. For this kind of analysis, the storm patterns for specified durations are presented in a dimensionless form with the relative rainfall depth (cumulative depth relative to total depth) as a function of time. With such a presentation, it is possible to perform the frequency analysis of the relative rainfall depth at the fixed times, and to construct a dimensionless storm pattern of a certain probability of occurrence (for details see Vukmirovic and Despotovic, 1984). These storm patterns are then used in conjunction with the IDF curves to obtain the design storms. Among the temporally variable design storms, the patterns of the "advanced" shape produced the most significant simulated peak flows.

\section{RESULTS}

Calculated frequencies of peak flow at the catchment outlet for the whole set and all subsets of historical storms are presented in Fig. 4. For all return periods above 2 years, observed flows are always greater than simulated flows from any set of historical storms. The set of all historical storms, the subset of storms with maximum intensity greater than $0.8 \mathrm{~mm} / \mathrm{min}$ and the subset of storms with depth greater than $10 \mathrm{~mm}$ give practically the same results (the first two overlap in the figure). This may lead to the conclusion that a set of the heaviest storms chosen on the basis of combined criteria (significant rainfall depth and intensity) could provide a reliable set of storms for simulation of most critical peak flows. However, this set should not be reduced to a very small number of events, because that would prevent a reliable frequency analysis of simulated flows. Moreover, this frequency analysis should then be based on the partial duration series approach rather than on the annual maxima series.

Fig. 4 also presents design flows obtained by rainfall-runoff simulation with three different types of design storms. The block design storms taken from the local IDF curves (what would be a common engineering practice) severely underestimate design flows. This is the consequence of underestimated rainfall intensities for shorter durations due to the insufficient rainfall record length. The block design storms from the neighbour rain-gauge station with a longer record perform slightly better, but still produce significant underestimation in the design flow frequencies. Introduction of the temporal variability of design storms improves the final output. With this approach, the design flows for return period of 5 and more years are underestimated for about $10 \%$ when compared to the observed flows, while there is a slight overestimation for the 2-year design flow.

The most obvious discrepancies are between the observed flows and the flows from the short-duration storms (up to 60 minutes). This extraordinary underestimation is not very surprising, because the observed storms of such a short duration rarely have significant total depth. Observed storms that produced the six highest peak flows are presented in Fig. 5 (with rainfall depths in time relative to total rainfall depth). All these storms consist of a short interval with a very high intensity and the remainder with weaker intensity. 
The real storm of a very short duration (say 15-20 minutes) does not have enough time to "develop", i.e. to have both the interval of a very high intensity and sufficient total depth. Fig. 5 also shows that rainfall temporal pattern of the most intense storms can be either advanced or delayed, but it is never close to uniform intensity, which is one of the most usual assumptions when design storms are taken directly from IDF curves.

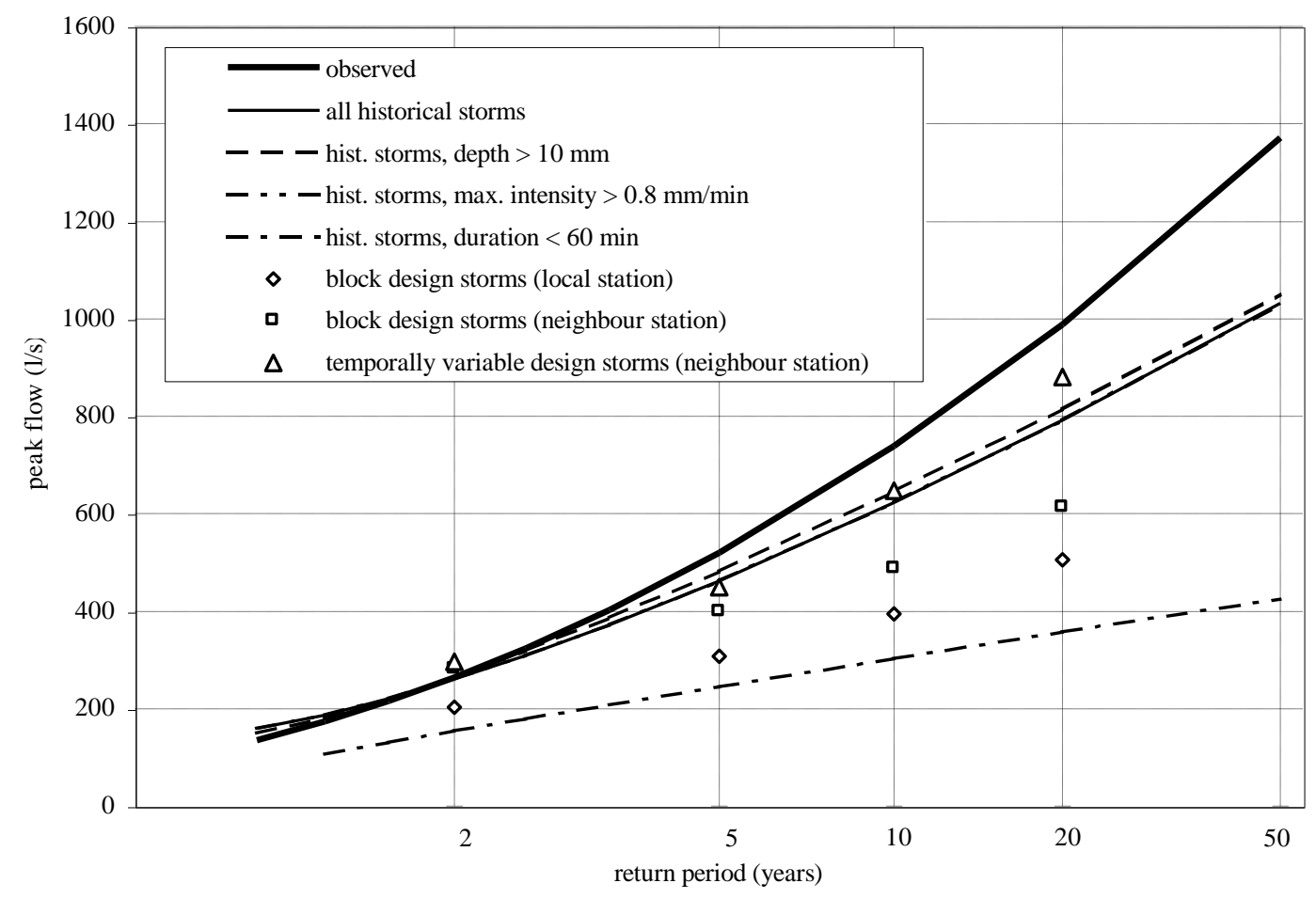

Figure 4. Frequencies of peak flows: observed flows and flows simulated with various sets of historical storms and with design storms.

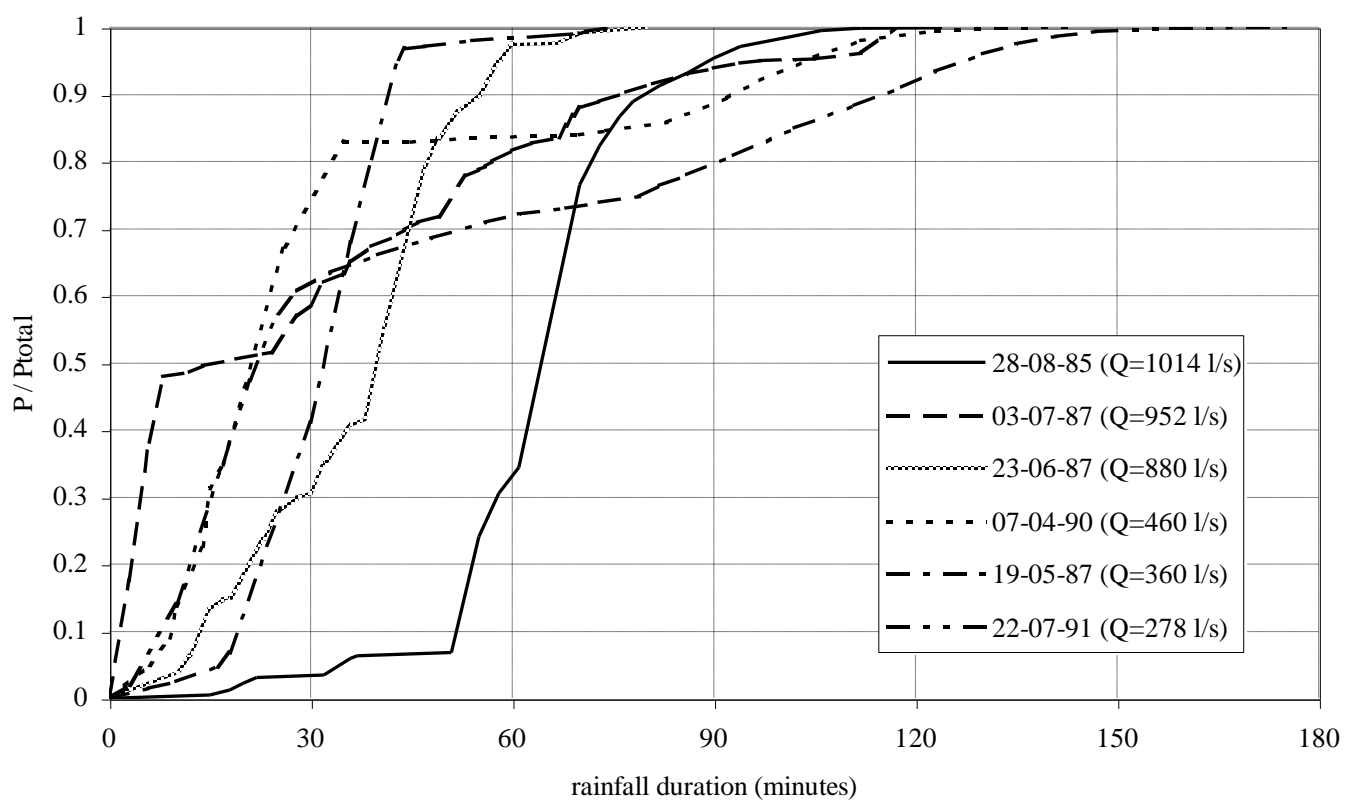

Figure 5. Relative cumulative rainfall depth for six storms that generated six greatest peak flows at catchment outlet. 


\section{CONCLUSIONS}

The possibilities of reducing the set of historical storms to be involved in realistic flow frequencies estimation was considered. The first conclusion is that any set of historical storms, fed to rainfall-runoff model, gives underestimated peak flows for return periods greater than 2 years (compared to observed peak flows). However, this underestimation may not be serious if a chosen set of historical storms represents the heaviest storms with regard to rainfall depth and intensity. This set of storms should not consist of a small number of events, because that would prevent reliable frequency analysis of simulated flows. Frequency analysis should be performed on partial duration series rather than on annual maxima series.

The assumption that the design set of historical storms should consist of storms with duration close to catchment's time of concentration leads to the entirely unrealistic results. Inspection of events that generated the highest observed peak flows at the experimental catchment proved that rainfall duration should not be considered as criterion for storm selection. Very short isolated storms may have high intensity, but they cannot reach significant depths.

The traditional approach with design storms taken from IDF curves as the block storms gives unacceptable underestimation of design flows, while the application of the temporally variable design storms produces results comparable with the frequencies of observed flows.

A question is also raised whether the main rainfall variable should be rainfall depth or rainfall intensity (the first reflects the largest water quantities, and the second one the most severe storms). Rainfall intensity plays an important role in the generation of extreme flows (Fig. 2): the higher intensity, the greater possible peak flow, but other factors (such as total rainfall depth, antecedent conditions, contribution of pervious area) limit the realistic value of peak flows. However, these conclusions about rainfall characteristics relevant for peak flows could not be transferred to other effects in the stormwater system (runoff volume, combined sewer overflows, retention inflows). Such problems should involve analysis of different rainfall characteristics and analysis of other variables, such as runoff volume, inter-event time, downstream conditions, and sediment wash-off and deposition.

\section{REFERENCES}

Despotovic J., Petrovic J. and Vukmirovic V. (1996). Some considerations of urban drainage design practice using experimental data. Atmos. Res., 42(1-4), 279-292.

Despotovic J. and Petrovic J. (1996). Measured peak flows at experimental catchment and flows estimated from various rainfall input. Proc. 7th Int. Conf. on Urban Storm Drainage (ed. F. Sieker and H.-R. Verworn), Hannover, pp. 1617-1622.

Harremoës P., Jensen M. and Johansen N. B. (1984). A staged approach to application of rainfall data to urban runoff calculations. Wat. Sci. Tech., 16(8/9), 237-250.

Maksimovic C., Despotovic J., Trisic P. and Simic M. (1986). Accuracy and reliability of rainfall and runoff measurements - examples, Proc. Int. Symp. on Comparison of Urban Drainage Models with Real Catchment Data, Supplements (ed. C. Maksimovic and M. Radojkovic), Dubrovnik, Yugoslavia, pp. 127-133.

Packman J. C. and Kidd, C. H. R. (1980). A logical approach to the design storm concept. Water Resources Research, 16(6), 994-1000.

Radojkovic M. and Maksimovic C. (1984). Development, testing and application of Belgrade urban drainage model, Proc. 3rd Int. Conf. on Urban Storm Drainage, Göteborg, Vol. 4, pp. 1431-1443.

Vukmirovic V. and Despotovic J. (1984). Statistical methods of storm analysis. Wat. Sci. Tech., 16(8/9), $85-92$.

Zhu H., Tveit O. A., Heuberger J., Wirth H. and Schilling W. (1996). Derivation of design storm by longterm simulation in a large sewer system. Proc. 7th Int. Conf. on Urban Storm Drainage (ed. F. Sieker and H.-R. Verworn), Hannover, pp. 13-18. 